\title{
Research on the retrieval of rain rate using polarimetric GNSS signals
}

\author{
An Hao*,Yan Wei,Bai Heng,Liu Wenjun \\ College of Meteorology and Oceanography \\ PLA University of Science and Technology \\ Nanjing, China \\ *excellentboy1990@gmail.com
}

\author{
Fu Yang \\ Beijing Institute of Applied Meteorology \\ Beijing, China \\ fuyang@shao.ac.cn
}

\author{
Han Ying \\ Beijing Institute of Petrochemical Technology \\ Beijing, China \\ hying@bipt.edu.cn
}

\begin{abstract}
In recent years, the Atmosphere-Ocean and Space environmental remote sensing technology such as atmospheric temperature and moisture profiles, sea surface wind field, and total electron content based on the GNSS signals has been widely concerned. This paper researches the mechanism and method of the retrieval of rain rate based on the depolarization of GNSS signals. According to a non-spherical raindrops model and raindrop size distribution, the function relation between the cross-polarization discrimination XPD defined to describe the depolarization and rain rate is established based on the non-spherical particle scattering algorithm. The feasibility of retrieving rain rate using the XPD is simulated and analyzed. The new technology of real-time, continuous, global detection of rain rate explored in this paper is of great significance to the expansion of the application of GNSS signals in the field of remote sensing of the atmosphere-ocean environment.
\end{abstract}

Keywords-polarimetric GNSS signals; rain rate; crosspolarization discrimination XPD

\section{INTRODUCTION}

Currently, the applications of Global Navigation Satellite System (GNSS) are becoming more and more extensive and popular. Today, GNSS comprises four major constellations: the United States' Global Positioning System (GPS), the Russian Federation's Global Orbiting Navigation Satellite System (GLONASS), Europe's Galileo, and China's BeiDou or Compass Navigation Satellite System ${ }^{[1]}$.However, the last two systems are still being deployed. The GNSS defined as the "worldwide position and time determination system" can be used in many regions such as navigation, surveying and mapping, agriculture, marketing, social networking, earth sciences, atmospheric sciences etc.Along with the development of GPS meteorology and GNSS-R, many meteorological and oceanographic elements such as precipitable water vapor, temperature profile and sea surfaces winds can be detected from GNSS signals ${ }^{[2][3][4]}$. Each GNSS satellite transmits a right hand circularly polarized(RHCP) signal at frequencies from $1.164 \mathrm{GHz}$ to $1.6155 \mathrm{GHz}$ which belongs to L-band ${ }^{[5]}$. Table I provides the center frequencies of GNSS signals ${ }^{[5][6]}$.

TABLE I. CENTERED FREQUENCIES OF GNSS SIGNALS (MHZ)

\begin{tabular}{|c|c|c|c|}
\hline GPS & GLONASS & Galileo & BeiDou \\
\hline $1575.42(\mathrm{~L} 1)$ & $1602.0(\mathrm{~L} 1)$ & $1575.42(\mathrm{E} 1)$ & $1589.74(\mathrm{E} 1)$ \\
\hline $1227.60(\mathrm{~L} 2)$ & $1246.0(\mathrm{~L} 2)$ & $1191.795(\mathrm{E} 5)$ & $1561.10(\mathrm{E} 2)$ \\
\hline $1176.45(\mathrm{~L} 5)$ & - & $1278.75(\mathrm{E} 6)$ & $1268.52(\mathrm{E} 6)$ \\
\hline- & - & - & $1207.14(\mathrm{E} 5 \mathrm{~b})$ \\
\hline
\end{tabular}

Rain is an important and recurrent phenomenon in meteorology which has a serious effect on the communication links, especially the earth-space link. Many papers had researched the rain attenuation and depolarization that are proportional to rain rate ${ }^{[7][8]}$. At L-band, the rain attenuation can be neglected, for the reason that although the rain rate is $150 \mathrm{~mm} \mathrm{~h}^{-1}$, it's still less than $0.1 \mathrm{~dB} \mathrm{~km}^{-1}{ }^{[9]}$. But, the raininduced depolarization across the earth-space rain path is strongly connected with rain rate. R.R.TAUR had measured the rain depolarization on a satellite-earth propagation path at 4 $\mathrm{GHz}^{[8]}$. However, the research on the rain depolarization at Lband is rarely. Furthermore, the method that using rain depolarization of GNSS signals for the retrieval of rain rate hasn't been studied.

Traditionally, the rain rate is directly measured by rain gauge which is of a tipping-bucket type. Radar reflectivity factor $(Z)$ is also used to estimate rain rate (R). And, radar polarimetric methods for rain rate measurements have received increasing attention in recent years. The specific differential phase (KDP) has a strong relation to rain rate $(\mathrm{R})^{[10]}$. In addition, some spaceborne instruments such as the Special Sensor Microwave Imager (SSM/I), the precipitation radar (PR) flying aboard the U.S.-Japanese Tropical Measuring Mission 
(TRMM) satellite and the microwave instrument (TMI) can yield information about rainfall ${ }^{[11]}$. However, those approaches are known to have limited accuracy and relatively coarse spatial resolution. While utilizing polarimetric GNSS signals to estimate rain rate has a high spatial resolution.

This paper has calculated the relationship between the rain depolarization and rain rate at the frequencies of GPS signals. The feasibility of the retrieval of rain rate based on rain depolarization has been discussed.

\section{METHODS}

\section{A. the Cross-polarization Discrimination XPD}

Rain depolarization is caused by the nonspherical shape of raindrops; spherical drops do not cause depolarization. In fact, during rainy conditions, due to the resistance of the air, surface tension, hydrostatic pressure and aerodynamic pressure, the raindrops aren't always spheres. A radio is used to measure the degree of depolarization, the cross-polarization discrimination XPD, defined as ${ }^{[12]}$ :

$$
X P D=20 \lg \left|\frac{E_{11}}{E_{12}}\right|
$$

where $E_{11}$ represents electric field intensity of copolarized wave and $E_{12}$ represents field intensity of crosspolarized wave. For example, for GNSS signals, the field intensity of the Right-Hand Circular Polarization wave is expressed as $E_{11}$, and that of the Left-Hand Circular Polarization wave as $E_{12}$. The radio XPD is expressed in $\mathrm{dB}$.

Considering the polarization of GNSS signals, the raininduced XPD of a Right-Hand Circular Polarization wave on earth-space links can be obtained from the following expression ${ }^{[13]}$ :

$$
X P D_{R}=20 \lg \left|\frac{(1+G) e^{2 \phi i}}{1-G}\right|,
$$

where, $G$ is only an intermediate variable, which is related to free-space propagation constant $k$, the forward scattering amplitudes $f_{h}$ and $f_{v}$, the rain drop size distribution $n(r)$.

Therefore, in order to achieve the $X P D_{R}$, the forward scattering amplitudes in the horizontal and vertical directions due to a single raindrop and the rain drop size distribution are necessary. The raindrop shape model, the complex dielectric constant of water and the non-spherical particle scattering algorithm are essential to calculate the forward scattering amplitudes ${ }^{[13] .}$

\section{B. Raindrop Shape Model}

The raindrops are often considered as spheres in study. However, small raindrops appear to have the shape of a sphere; while larger raindrops become increasingly deformed from spheres resulting in ellipsoid-like shapes with a flattened base. Previously, the oblate spheroid model was studied. In order to describe the ratio of the minor to major axes of oblate spheroid drops, a nonlinear equation is often used ${ }^{[14]}$ :

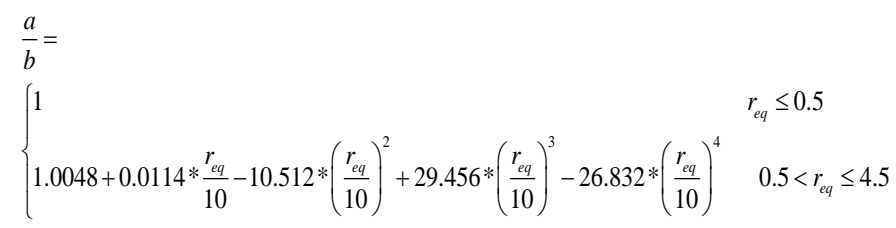

where, $r_{e q}$ is the radius of a spherical raindrop having the same volume with the spheroidal raindrop in millimeters. Figure 1 shows the shapes of the oblate spheroid raindrops with axial ratio by Eq.3.The shapes model is similar with the typical Modified Pruppacher-and-Pitter model developed by Pruppacher and Pitter to describe the realistic raindrop shapes [15].
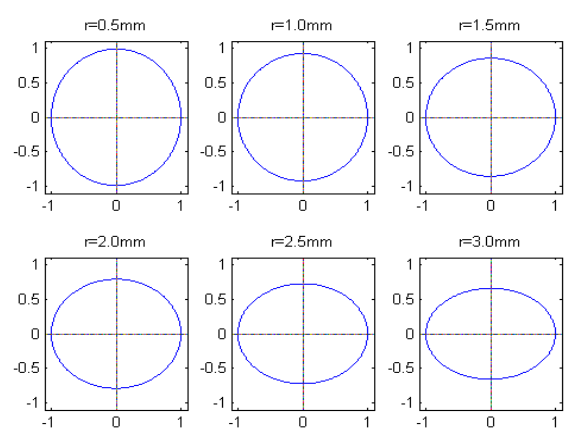

Fig. 1. Normalized oblate spheroid raindrop shapes with axial ratio given by Eq.3

\section{Drop Size Distribution}

Drop size distribution (DSD) refers to the number-size distribution of raindrops per volume. An accurate simulation result of XPD requires detailed knowledge of rain drop size distribution. Currently, the model linking the rain rate with drop radius is commonly assumed to be a negative exponential function as proposed by Marshall and Palmer [13]. The following empirical relation was suggested:

$$
N(r)=N_{0} \exp (-\Lambda r)
$$

where

$$
\begin{aligned}
N_{0} & =1.6 \times 10^{4} & & \left(\mathrm{~m}^{-3} \mathrm{~mm}^{-1}\right), \\
\Lambda & =8.2 R^{-0.21} & & \left(\mathrm{~mm}^{-1}\right),
\end{aligned}
$$

and where $r$ is the equivalent radius of a raindrop in millimeters and $R$ is rain rate in millimeters per hour.

\section{Complex Dielectric Constant of Water}

The complex dielectric constant of water is needed in scattering algorithm. It's often expressed as $\varepsilon\left(\varepsilon=\varepsilon^{\prime}-i \varepsilon^{\prime \prime}\right)$, where $\varepsilon^{\prime}$ is the dielectric constant and $\varepsilon^{\prime \prime}$, the loss. The complex dielectric constant depend not only frequency but also on temperature. An empirical model of the complex dielectric constant of water was obtained by Ray [16], which is suitable over a large portion of the spectrum and for a wide range in temperature. 


\section{E. Non-spherical Particle Scattering Algorithm}

Due to the fact that the raindrops aren't spherical, the classic Mie scattering calculating model isn't suitable here. Many authors have developed some theory to solve the problem of non-spherical particle scattering. Mainly, the scattering algorithms for non-spherical particles are Rayleigh approximation, T-matrix, Point Matching, Boundary Element Method, Unimoment, and Fredholm Integral Equation ${ }^{[13][17][18]}$.

Because the frequencies of GNSS signals are from $1.164 \mathrm{GHz}$ to $1.6155 \mathrm{GHz}$, which is in L-band, then the wavelengths of GNSS signals can be calculated, which are between $18.57 \mathrm{~cm}$ and $25.77 \mathrm{~cm}$. It's obvious that the wavelengths are much larger than the radii of raindrops $(0.25 \sim 4.5 \mathrm{~mm})$. Therefore, the scattering algorithm Rayleigh scattering approximation is feasible.

\section{RESULTS AND ANALYSIS}

In the model, Ray's formula at the water temperature of $20^{\circ} \mathrm{C}$ was used to calculate the complex dielectric constant of water. Here, we take the frequencies of GPS signals for example. From Table I, the L1, L2 and L5 are used. The elevation of GPS satellite was assumed $0^{\circ}$, at which the degree of depolarization is probably much serious ${ }^{[19]}$. The raininduced XPD is described as a function of rain rate in Fig.2. And Fig.2a, Fig.2b and Fig.2c are rain-induced XPD of L1, L2 and L5, respectively.

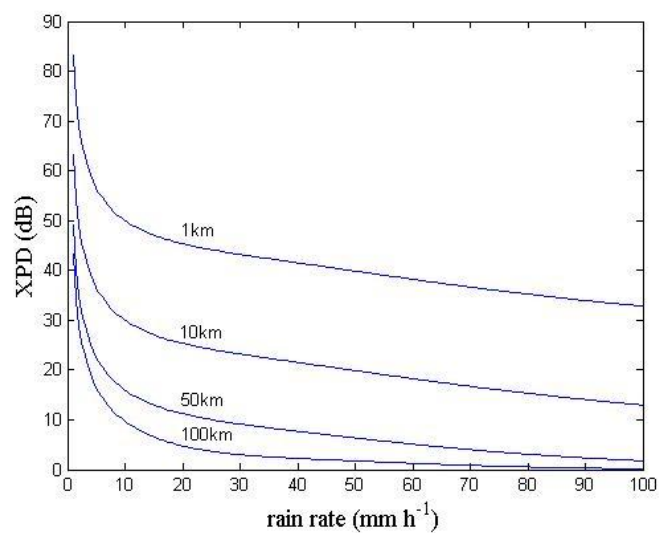

(a)

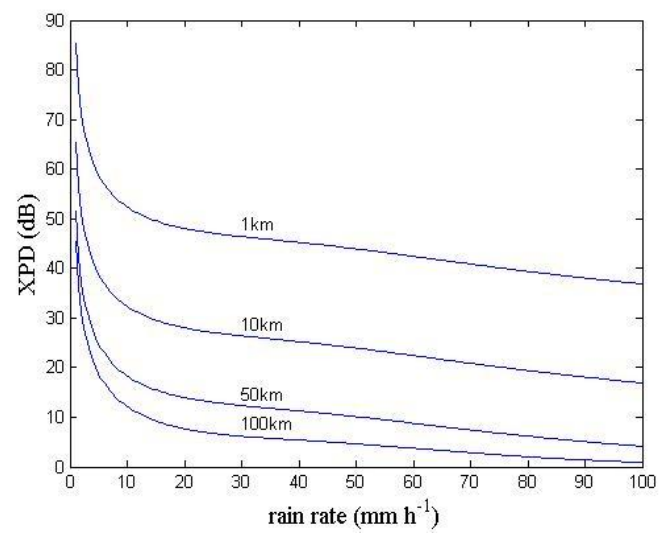

(b)

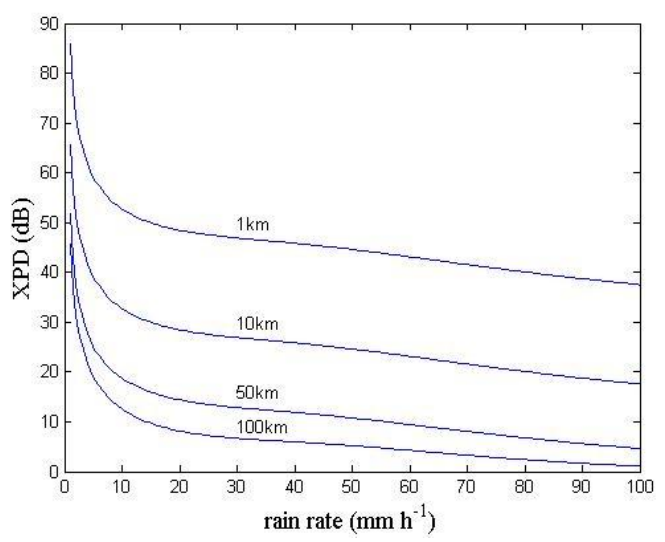

(c)

Fig. 2. The simulated relationship between the rain-induced XPD and rain rate at different path lengths and frequencies of GPS signals, (a) at the frequency L1; (b) at the frequency L2; (c)at the frequency L5

From Fig.2, it can be seen that all the lines have a consistent trend, even if at different path lengths. When the rain rate is increasing, the rain-induced XPD decreases smoothly. In addition, we also note that the XPD's tendency is similar at different frequencies of GNSS signals. And the XPD reduces obviously along with the path lengths of GNSS signals, which indicates that the longer path length causes more serious depolarization. The results are in concord with the research by R.R. TAUR ${ }^{[8]}$.

Most important of all, the Fig.2 shows that the rain-induced XPD of GNSS signals is sensitive to rain rate, which isn't contrary to the results by Oguchi ${ }^{[13]}$. The rain-induced XPD has the feasibility of the retrieval of rain rate.

\section{CONCLUSIONS}

This paper has investigated the effect of depolarization caused by rain and calculated the rain-induced XPD of GNSS signals. The relationships between XPD and rain rate at different path lengths and frequencies of GNSS signals are simulated and the feasibility to estimate rain rate is analyzed. The results have indicated that the theory has its potential to retrieve rain rate.

Furthermore, the rapid development of the establishment of the GNSS network makes it more convenient to detect rain rate. This new technique for retrieving rain rate has its potential applications in global precipitation reconnaissance and weather forecast. And specific experimental program is being designed to study its practicability in the following researches.

\section{REFERENCES}

[1] Christopher J. Hegarty, "GNSS Signals - An Overview," 2012 IEEE.

[2] Jingjing Xu, Bo Liu, Jinguo Yuan, Changyao Wang, "Inversion of Precipitable Water Vapor in Hongkong," 2008 IEEE.

[3] M Armatys. "Estimation of Sea Surface Winds Using Reflected GPS Signals," $\mathrm{PhD}$ thesis, University of Colorado at Bolder, 2001. 
[4] T. P. Yunck, "An Overview of Atmospheric Radio Occultation," Journal of Global Positioning Systems Vol. 1, No.

[5] Sarang Thombre, Heikki Hurskainen, Jari Nurmi, "Wideband, High Gain, High Linearity, Low Noise Amplifier for GNSS Frequencies with Compensation for Low Frequency Instability,” 2008 IEEE, pp: 349-354.

[6] Grace Xingxin Gao, Alan Chen, Sherman Lo, David De Lorenzo, Todd Walter, and Per Enge, "Compass-M1 Broadcast Codes in E2, E5b, and E6 Frequency Bands," IEEE JOURNAL OF SELECTED TOPICS IN SIGNAL PROCESSING, Vol. 3, No. 4, pp: 599-612, 2009.

[7] Ali, A., M. Hassan, and M. Alhaider, "Experimental Studies of Terrestrial mm-Wave Links-A Review Part 1: Attenuation Due to Atmospheric Particles,” J. Eng. Sci., Vol. 12, No. 2, pp: $197-$ 212, 1986

[8] R. R.TAUR, "Rain Depolarization Measurements on a SatelliteEarth Propagation Path at 4 GHZ," IEEE Trans. Antennas Propag., Vol.23, pp:854-858,1975.

[9] ITU-R P.838-3, "Specific attenuation model for rain for use in prediction methods, 1-8, 2005

[10] S.Y. Matrosov, R.A. Kropfli, B. E. Martner, and R.F. Reinking, "X-band estimations of rainfall with differential propagation phase shift measurements," Geoscience and Remote Sensing Symposium Proceedings, Vol.1, pp: 446-448, 1998.

[11] Kawanishi1, T., H. Kuroiwa, and M. Kojima, "TRMM Precipitation Radar," Adv. Space Res., Vol.25, pp:969-972, 2000 .
1, pp: 58-60, 2002.

[12] W.L. Flock, "Propagation effects on satellite systems for frequencies below $10 \mathrm{GHz}$," NASA Ref. Publ., Vol.1108, pp: 1510, 1987.

[13] Oguchi T., "Electromagnetic Wave Propagation and Scattering in Rain and Other Hydrometeors," Proc. IEEE, Vol.71, pp: 1029-1079, 1983

[14] Bahrami, M., J. R. Mohassel, and M. M. Taheri, "An exact solution of coherent wave propagation in rain medium with realistic raindrop shapes," Progress In Electromagnetics Research, PIER79, pp: 107-118, 2008.

[15] Li, L.W., P. S. Kooi, M. S. Leong, T. S. Yeo, and M. Z. Gao, "Microwave Attenuation by Realistically Distorted Raindrops: Part I-Theory," IEEE Trans. Antennas Propag., Vol.43,pp:811$822,1995$.

[16] Peter S. Ray, "Broadband Complex Refractive Indices of Ice and Water," Appl. Opt., Vol.11, pp: 1836-1844, 1972.

[17] Mishchenko, M.I., and L. Travis, "Capabilities and limitations of a current FORTRAN implementation of the T-matrix method for randomly oriented, rotationally symmetric scatterers," J. Quant. Spectrosc. Radiat. Transfer, Vol.60, pp: 309 -324, 1998.

[18] [18] Duffo, N., and M. Vall-llossera, A. Camps, I. Corbella, and F. Torres, "Polarimetric Emission of Rain Events: Simulation and Experimental Results at X-Band," Remote Sens., Vol.1, pp: 107-121, 2009.

[19] E. Cardellach, A. Rius, and F. Cerezo, "Polarimetric GNSS Radio-Occultations for heavy rain detection, Geoscience and Remote Sensing Symposium,” pp: 3841-3844, 2010. 